\title{
Recovery of Deglutition Disorder in Posterior Inferior Cerebellar Artery Stroke-A Case Reports
}

\author{
K Govathi Nikhila*, Akanksha Gupta, Sushant Bhuyan and Arun Garg \\ Medanta- The Medicity Hospital, Department of Neurosciences, India
}

Submission: January 05, 2017; Published: January 13, 2017

*Corresponding author: K Govathi Nikhila, TRT122/A, Seethaphalmandi, Secunderabad, Telangana, 500061, Tel: 9582972948;

Email: gnikhilaks@gmail.com

\begin{abstract}
Introduction: Posterior Inferior Cerebellar Artery stroke is most commonly seen with dysphagia, dysarthria, and gait problems. In this we are presenting 2 patients who received combined Traditional therapy and vital stim therapy and only swallow rehabilitation therapy along with the recovery pattern.

Methods: A patient with 63 years and 65 years old Men were reported to our hospital with a stroke, with a common history of Hypertension, and non-diabetic, and with sudden onset of dysphagia, dysarthria and ataxia of the gait, but one person was smoker. The patients was clinically diagnosed with a acute ischemic stroke with left posterior inferior cerebellar artery infarct .Clinical dysphagia bed side evaluation was done along with FEES test and graded with level1 based on their National Outcome Measurements system (NOMS). Swallowing level scale was used as parameter for evaluating the level-1.Later, one the patient has received combined vital stim therapy with traditional therapy for number of sessions where as other not.
\end{abstract}

Results: Deglutition disorder was clinically more severe in patients with PICA syndrome than in other groups. The pharyngeal phase of swallowing was predominantly impaired .Fast recovery has seen with combination of vital stim therapy and traditional therapy and the patient was graded with level-7 based on National outcomes Measurements system (NOMS).

Conclusion: combined vital stim electrical therapy and traditional therapy has greater effect to overcome dysphagia. However, large placebo randomised study is required to establish the effective recovery patterns.

Abbreviations: PCS: Posterior Circulation Stroke; PICA: Posterior Inferior Cerebellar Artery; NOMS: National Outcomes Measurements system; VB: Vertebrobasilar; FEES: Fiberoptic Endoscopic Evaluation of Swallowing; VST: Vital Stim Therapy; MRI: Magnetic Resonance Imaging; CT: Computed Tomography; RTF: Ryle's Tube Feeding; NG: Nasogastric Tube; NJ: Nasojejunal Tube

\section{Introduction}

A posterior circulation (PC) stroke is classically defined by infarction occurring within the vascular territory supplied by the vertebrobasilar (VB) arterial system. Posterior circulation strokes represent approximately $20 \%$ of all ischemic strokes [1$3]$. In contrast to the anterior circulation, several differences in presenting symptoms, clinical evaluation, diagnostic testing, and management strategy exist presenting a challenge to the treating physician. A constellation of neurological features, resulting from stroke in the vertebral or posterior inferior cerebellar artery of the brain stem, constitute Wallenberg syndrome [4]. It is also called as lateral medullary syndrome or posterior inferior cerebellar artery syndrome. The lateral region of the medulla oblongata presents the anterior lateral sulcus ventrally and the posterior lateral sulcus in the dorsal aspect. The pons flanks it rostrally and the cervical spinal cord caudally. Multiple nerve nuclei and tracks are found in this region. Some of them are- the inferior cerebellar peduncle, vestibular nuclei, spinal trigeminal nucleus, descending sympathetic fibers, dorsal motor nucleus of the vagus nerve, lateral spinothalamic tract, nucleus ambiguous, solitary nucleus, medullary reticular formation, and central tegmental tract.

Due to compact placement of nuclei and tracts in the lateral aspect of the medulla, clinical features of Wallenberg syndrome result due to abnormalities in the vestibulo-cerebellar, sensory, bulbar, respiratory, and autonomic systems [5,6]. In case of ipsilateral deglutition disorder, hoarseness and poor gag reflex, the injury involves nucleus ambiguous. This is located relatively 


\section{Global Journal of Otolaryngology}

medial in the lateral medullary region and is responsible for functioning of vocal cord and palatal muscles function. Ipsilateral Horner's syndrome (miosis, ptosis, and anhidrosis) occurs when damage to descending sympathetic fibers occurs. In certain patients, disguise (distortion of taste) occurs, which is due to involvement of solitary nucleus [4-8]. Tachycardia, orthostatic hypo-tension without tachycardia and intermittent bradycardia may be found if the dorsal motor nucleus of the vagus nerve is affected, due to involvement of the autonomic system. Undine's curse or failure of automatic respiration leading to respiratory arrest during sleep may occur in injury to the ventrolateral medullary tegmentum and medullary reticular zone [3-8] (Figure 1).
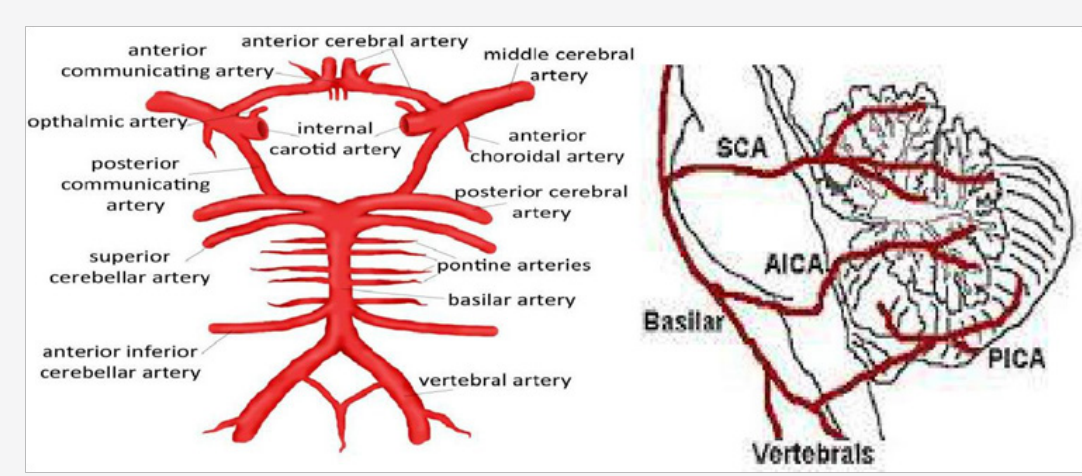

Figure 1: Vertebrobasilar system.

\section{Case -1}

\section{Case details}

A. Patient history information: The patient is 63 year old male brought to Medanta Hospital on 26/09/15 with a past history of Hypertension and non-diabetic and with chief Table 1: Case I- Investigations. complaint of sudden onset of dizziness 4 days back while bathing followed by facial deviation, difficulty in walking and difficulty in swallowing. Then patient was immediately took to the local nearby hospital and NCCT head was done and suggestive old infarct in right parieto-occipital region. Then he brought to the Medanta after 4 days for further evaluation (Table 1).

\begin{tabular}{|c|c|c|}
\hline Present Medical History & Review of Systems & Neurological \\
\hline Hypertension & \multirow{4}{*}{$\begin{array}{l}\text { Nasal congestion, mild nonproductive } \\
\qquad \text { cough } \\
\text { Mild dizzy spells for past } 4 \text { days each } \\
\text { Lasting 5-10 minutes. }\end{array}$} & \multirow{16}{*}{$\begin{array}{c}\text { Mental status: Patient Conscious } \\
\text { Congenital squint right eye } \\
\text { Speech -Dysarthria present } \\
\text { Reduced palatal movement } \\
\text { Weak gag reflex } \\
\text { Left Cerebellar signs present } \\
\text { Power left UL } 4 / 5 \\
\text { Planters left up going and right down going. }\end{array}$} \\
\hline $\begin{array}{c}\text { Seizures, generalized, none in past } 7 \\
\text { years }\end{array}$ & & \\
\hline Risk factors & & \\
\hline Alcohol N/A & & \\
\hline Obesity N/A & Physical Exam & \\
\hline CAD N/A & Vitals & \\
\hline Valvular Heart disease N/A & \multirow{2}{*}{$\begin{array}{c}\text { Gen: Patient lying comfortably, in no apparent } \\
\text { distress }\end{array}$} & \\
\hline Atrial fibrillation N/A & & \\
\hline Homocysteinemia N/A & Pupils: R 3.5/ L 3.0 reactive. Fundi clear. & \\
\hline Dyslipidemia N/A & Chest: B/L Clear & \\
\hline Smokes 1 pack per day - 25years & \multirow{2}{*}{ Abdomen: Soft, nontender, no } & \\
\hline \multirow[t]{5}{*}{ No family history of stroke. } & & \\
\hline & GCS:E4V5M5-6 & \\
\hline & BP:140/90mm Hg & \\
\hline & Pulse:88/min & \\
\hline & $\begin{array}{l}\text { Temp: Afebrile } \\
\text { CVS: S1,S2 Normal }\end{array}$ & \\
\hline
\end{tabular}


B. MRI/CT scanning was done on (26/09/15) Impression of CT Head: Hypodense area is seen in left posterolateral medulla, posterioinferior cerebellum consistent with acute evolved infarcts in Left PICA territory.

C. MRI brain with MR Angiography of neck and brain vessels was done and Impression was Acute Left PICA territory infarcts involving left cerebellar hemisphere, posterolateral medulla, and inferior cerebellar hemispheres. Complete occlusion of left vertebral artery from its origin through its course in the neck and cranial cavity. No other significant vascular stenosis.

D. ECHO- was done on $(26 / 09 / 15)$ and results revealed No LV RWMA, LVEF-55\%.Normal RV systolic function. Normal all cardiac chamber dimensions. MIP-DRA. Normal LVEDP. PASP-26 mm of Hg. NO AS/AR. Normal IVC, CVP-3mmof Hg. No I/C clot/veg/PE.

E. X-RAY CHEST: was done 27/9/15 and findings are Rotation++ medical lines and tubes are noted. Haziness is seen in left lower zone and left CP angle suggestive of left pleural effusion with underlying atelectasis. Blunting of right $\mathrm{CP}$ angle is noted suggestive of right pleural effusion. Rest of lung field appears normal .cardiac size cannot be commented upon.

F. NCCT BRAIN: was done on $(01 / 10 / 15)$.clinical details: case of posterior circulation stroke. Comparison has done with the previous MRI brain dated 26/09/15and Findings reveals: no significant interval changes in the wedge-like area of acute to sub-acute left PICA territory infarct in cerebellar hemisphere, inferior vermis and left posterolateral medulla.

\section{Rehabilitation Management}

i. Deglutition Assessment and Therapy: The patient was received Initial Bedside swallow Evaluation on the day 5 of his admission and fiberoptic endoscopic evaluation of swallowing (FEES) was done on next day and results reveals Aspiration present then, later, patient was graded with Level-1 based on the National Outcome Measurements system(NOMS). Then patient is recommended to continue the Ryles tube Feeding (NJ) and Swallow therapy. This patient has received combined swallow therapy along with vital stim electrical stimulation from the day 5. The patient has been reviewed with re-swallow evaluation for every 3-4 days and graded with the levels based on NOMS.

Combined swallow therapy was received by the patient for 60 mins for 6 days in a weak. The patient have been received combined swallow therapy in IPD for 2 weeks and followed up for the same on OPD Bases. Later, After 24 days with 21 sessions of combined swallow therapy the patient has been started with oral diet with Semi solid food along with RTF and Graded with Level-3 Based on NOMS. On the day of 51 with 45 sessions the patients was started complete oral diet with Minimal soft diet along with compensatory strategies and graded with Level-5 based on NOMS. After a number of 63 sessions with number of 67 days the patient was Received Clinical swallow Evaluation along with FEES test and recommended for Normal Diet and graded with Level-7 based on NOMS.

II. Case Conclusion: This patient has received combined swallow therapy for 2 weeks in IPD bases and later continued with the same on OPD bases for 67 days and reached Level-7 I.e. Normal diet based on NOMS.

\section{Case -2}

\section{Case Details}

A. Patient History Information: The patient is 65 year old male brought to the Medanta Hospital on 26/01/16 with a past history of Hypertension and diabetic Mellitus typeII and with chief complaint of sudden onset of right side weakness associated with slurred speech and difficulty in swallowing at $6 \mathrm{pm}$ on $25 / 01 / 16$. Then patient was taken to local hospital and NCCT Head was done suggestive of no bleed/MLS, then later, patient was brought to Medanta hospital for further management (Table 2).

Table 2: Case II- Investigations.

\begin{tabular}{|c|c|c|}
\hline $\begin{array}{c}\text { Present Medical } \\
\text { History }\end{array}$ & Physical Exam & \\
\hline $\begin{array}{c}\text { Hypertension } \\
\text { Diabetes Mellitus } \\
\text { Type II }\end{array}$ & Vitals & Neurological \\
\hline $\begin{array}{c}\text { Diabetes Mellitus } \\
\text { Type II }\end{array}$ & BP:130/80mm Hg & On Examination \\
\hline Risk factors & Pupils: NSNR & \multirow{2}{*}{$\begin{array}{c}\text { Drowsy, arousable, } \\
\text { obeying shortly } \\
\text { GCS:E3V5M5-6 }\end{array}$} \\
\hline Alcohol N/A & Pulse:90/min & \\
\hline Obesity N/A & $\begin{array}{l}\text { Chest: Clear to } \\
\text { auscultation. }\end{array}$ & $\begin{array}{l}\text { Plantar: Right } \\
\text { extensor, Left flexor }\end{array}$ \\
\hline CAD N/A & $\begin{array}{l}\text { Heart: S1, S2 normal, } \\
\text { no murmurs. }\end{array}$ & \multirow{7}{*}{$\begin{array}{c}\text { Moving: Left>Right } \\
\text { side body } \\
\text { Right hemi neglect+ } \\
\text { Dysarthria + } \\
\text { Reduced palatal } \\
\text { movement } \\
\text { Poor gag reflex } \\
\text { Restricted lingual } \\
\text { upward movement }\end{array}$} \\
\hline $\begin{array}{l}\text { Valvular Heart } \\
\text { disease N/A }\end{array}$ & $\begin{array}{c}\text { Abdomen: Soft, } \\
\text { nontender, no }\end{array}$ & \\
\hline Atrial fibrillation N/A & organmegaly & \\
\hline $\begin{array}{c}\text { Homocysteinemia } \\
\text { N/A }\end{array}$ & & \\
\hline Dyslipidemia N/A & & \\
\hline Smoking & & \\
\hline $\begin{array}{c}\text { No family history of } \\
\text { stroke. }\end{array}$ & & \\
\hline
\end{tabular}

B. NCCT BRAIN was done on $(26 / 01 / 16)$ and results showed hypo density in left occipito-temporal cortex and white matter involving parahippocampal gyrus, mesiotemporal cortex suggesting acute evolved to sub-acute infarct in left PICA territory. Another hypodensity is noted in the subcortical whit matter of the right middle and inferior 
frontal gyri and external capsular region. Few old lacunar infarcts are seen in left gangliocapsular region and left thalamus.

C. CT brain plain and CT Angio brain and neck was done on (28/01/16) and results showed the sub-acute infarct in left PICA territory subacute infarcts are also noted in the subcortical white matter of the right middle and inferior frontal gyri and external capsule region.

D. NCCT BRAIN was again done on $(01 / 02 / 16)$ showed temporal evolution of sub-acute left PCA infarct is seen in occipito-temporal cortex and white matter involving parahippocampal gyrus, mesio-temporal region. No hemorrhage is seen in infarcted region hypodensity is noted in subcortical white matter of the right middle and inferior frontal gyri and external capsule region.

\section{Rehabilitation Management}

i. Deglutition Assessment and Therapy: The patient was received Initial Bedside swallow Evaluation on the day 5 of his admission and fiberoptic endoscopic evaluation of swallowing (FEES) was done on next day and results reveals Aspiration present then, later, patient was graded with Level-1 based on the National Outcome Measurements system (NOMS) .Then patient is recommended to continue the Ryles tube Feeding (NJ) and Swallow therapy. This patient has received combined swallow therapy along with vital stim electrical stimulation from the day 5.

The patient has been reviewed with re-swallow evaluation for every 3-4 days and graded with the levels based on NOMS. Combined swallow therapy was received by the patient for 60 mins for 6 days in a weak. The patient have been received combined swallow therapy in IPD for 3weaks-4 days and got discharged with level 2 i.e., patient can take some consistency of food (4-5 spoon of semi-solid blended food along with the compensatory swallow maneuver chin tuck was recommended while swallowing and the care taker was also educated about the same), as the patient home town was far so patient couldn't attended for combined swallow therapy on OPD Bases. But the patient and the care takers got educated about the Active swallow Exercises and provided with the hand-outs and recommended to do the exercises for $3-4$ times a day and each to be repeated for 10 times. Later, the patient was visited on OPD after 20 days for Swallow Evaluation then clinical swallow test along with FEES test was done and there was no Aspiration noted with semi solid food (custard) but with liquids mild aspiration was noted for which there was no response from the patient.

Then the patient was recommended with 3 times semi solid food orally along with compensatory swallow maneuver chin tuck along with RTF and graded with Level- 3 based on NOMS .Patient was also recommended to avoid solid, and liquids food orally and recommended to continue the swallow exercises.
Later, patient was visited for swallow evaluation after 20 days and the clinical swallow test along with FEES test was done with liquids and soft diet but still there was an Aspiration noted with Liquids. Then the patient was recommended with minimal RTF for fluid hydration and asked to Increase the Oral diet (soft diet) along with compensatory swallow maneuver chin tuck and graded with Level-4 based on NOMS and recommended to do intensive swallow exercises. Then the patient was visited on OPD after 10 days the clinical swallow evaluation along with FEES test was done and the results are same as the above.Hence, the patient was recommended to continue the same diet and recommended to do intensive swallow exercises.

After 20 days again the patient was visited for swallow evaluation, then the clinical swallow evaluation along with FEES test was done and there was no aspiration noted then the patient was recommended for complete oral diet (soft diet) along with compensatory swallow maneuver chin tuck with minimal food restrictions, and also asked to continue the exercises. On 3 months follow up through telephonic conversation the patient was graded with level-7 i.e., was taking normal diet orally.

II. Case conclusion: This patient has received combined swallow therapy for 3 weeks -4 days and later he continued only Active swallow exercises and followed up on OPD bases for swallow Evaluation after 96 days the patient has reached to Level -7 i.e. normal diets based on NOMS.

\section{Discussion}

The said syndrome was named after Adolf Wallenberg, renowned for his detailed neurological examination and focus on clinico-pathological correlation [3]. However, it was first described by Gaspard Vieusseux of Geneva, in 1810, at the Medical and Chirurgical Society of London as; "Vertigo, unilateral facial numbness, loss of pain and temperature appreciation in the opposite limbs, dysphasia and hoarseness, minor tongue involvement, hiccups (cured by taking up the habit of a morning cigarette) and a drooped eyelid" [4]. A clearer picture regarding this disease was obtained, in 1895, when Wallenberg himself established his findings which included postmortem results [3]. A Spanish study which reviewed 25 cases of lateral medullary syndrome found presentation by middle aged men at 55.06 years of age to be predominant. The most important risk factor was arterial hypertension. The onset was progressive in most cases. Features noted were dysphonia and dysphagia. Ataxic gate was found in majority of the patients. The clinical picture varied based on the exact location of the lesion. However, the prognosis was good in most cases with ataxia as a sequel.

Several anatomical and clinical differences distinguish PC infarctions from those in the AC and have practical implications. Vascular anatomical variation may affect stroke severity and presenting signs and symptoms. Large vessel atherostenosis is common but cardioembolic strokes, dissections, and other etiologies are known to affect the PC [9]. Quantitative measures 
such as the NIHSS alone may not sufficient and clinical signs and symptoms such as dizziness, vertigo, and ataxia may be diagnostic pitfalls. MRI is superior to CT for imaging an acute PC infarct but may rarely miss a small infarct early on. A variety of non-invasive vascular imaging modalities are available to aid the etiologic work-up and have replaced conventional catheter angiography in many circumstances. IV-rt-PA is the recommended first-line treatment for eligible patients.

In this we have represented two patients in which they both are meeting all the signs and symptoms of the PICA stroke and clinically and radiologically diagnosed as same. But among them both only one patient was received combined vital stim and swallow therapy were as the other one received only traditional swallow therapy [10]. The one who received combined swallow therapy have greater effect on dysphagia recovery were as the other has slow recovery pattern to overcome dysphagia.

\section{Conclusion}

Combination of Vital Stim Therapy and Traditional therapy has greater effect in recovery of dysphagia. However, large placebo control randomized one required to establish the recovery patterns.

\section{References}

1. Gulli G, Marquardt L, Rothwell PM, Markus HS (2013) Stroke risk after posterior circulation stroke/transient ischemic attack and its relationship to site of vertebrobasilar stenosis pooled data analysis from prospective studies. Stroke 44(3): 598-60410.

2. Labropoulos N, Nandivada P, Bekelis K (2011) Stroke of the posterior cerebral circulation. Int Angiol 30(2): 105-114.

3. Searls DE, Pazdera L, Korbel E, Vysata O, Caplan LR (2012) Symptoms and signs of posterior circulation ischemia in the new England Medical Center posterior circulation registry. Arch Neurol 69(3): 346-351.

4. Vladimir Kaye, Murray E Brandstater (2016) Vertebrobasilar Stroke Overview of Vertebrobasilar Stroke. Medscape.

5. Pearce JMS (2000) Wallenberg's syndrome. J Neurol Neurosurg Psychiatry 68(5): 570.

6. (1811) History of a singular Nervous or Paralytic Affection, attended with anomalous morbid sensations. Medico Chir Trans 2: 217-235.

7. Kim YK, Schulman S (2009) Cervical artery dissection: pathology, epidemiology and management. Thromb Res 123(6): 810-821.

8. Baugh CW, Brown DFM, Nadel ES (2009) Horner's syndrome, hoarseness, and unsteady gait. J Emerg Med 36(2): 176-180.

9. Kim JS (2003) Pure lateral medullary infarction: clinical-radiological correlation of 130 acute, consecutive patients. Brain J Neurol 126(Pt 8): $1864-1872$.

10. Sacco RL, Freddo L, Bello JA, Odel JG, Onesti ST, et al. (1993) Wallenberg's lateral medullary syndrome. Clinical-magnetic resonance imaging correlations. Arch Neurol 50(6): 609-611.

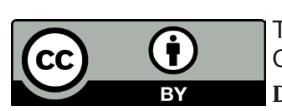

This work is licensed under Creative Commons Attribution 4.0 License DOI: $10.19080 / G J O .2017 .03 .555612$ 